\title{
Asymmetry in Health and Disease: Food for Thought
}

\author{
Okonkwo PO* \\ Department of Pharmacology and Therapeutics, University of Nigeria, Enugu, \\ Nigeria
}

*Corresponding author: Paul Obiekwe Okonkwo, Emeritus Professor

\section{Mini Review}

Volume 2 Issue 2

Received Date: September 19, 2018

Published Date: November 06, 2018

DOI: $10.23880 /$ eij-16000113

Department of Pharmacology and Therapeutics, University of Nigeria, College of Medicine, Enugu, Nigeria, Email: okonkwopaul6@gmail.com

\begin{abstract}
Asymmetry is the uneven or unequal distribution of physical attributes on the left and right sides of the body. There are certain diseases that show remarkable asymmetric distribution. The filarial infections in their superficial manifestations typify this asymmetry. Other anomalies such as facial and dental distortions, breast cancer, stroke, cryptorchidism all usually may manifest on one side of the body in the first instance. This short review is meant to provoke further research and discussions on this phenomenon. It does not present clear answers or solutions to this issue.
\end{abstract}

Keywords: Tropical diseases; Asymmetry; Filariasis

\section{Observations}

In the animal world, doubling of limbs was advantageous in movements. From the monkey gliding through the trees, to the tiger grabbing a prey by the jugular, dual limbs offer stability and balance. Even the kangaroo has stunted fore limbs but they are powerful and serve their purpose. Man does not have a tail, and in animals where tails occur, this organ is rarely replicated. Thus, organs are arranged symmetrically for function, not aesthetics in animals. In onchocerciasis or river blindness a filarial disease we have studied [1-4]. The vector Simulium damnosum can bite any part of the body to transfer the immature larvae of Onchocerca volvulus. These develop into adult worms. An average of two females and one female hide in subcutaneous benign tumors "nodules". A nodule can survive in the body for up to 15 years spewing out microfilaria. Nodules are more abundant in the pelvic region, but can be found in the headin children. The microfilaria can be detected in the body in skin snips, but the skin lesions are asymmetrically located in one limb. It is difficult to predict which limb will suffer de pigmentation, leopard skin or lichenfication. Handedness could not be studied as a factor of asymmetry because the use of the left hand is suppressed from infancy. Similarly, the onchodermattis will localize in one arm, the lesions in the back may be spread all over.

The extant drug for treatment of onchocerciasis is ivermectin which is a microfilariacide. Patients treated with ivermectin develop an asymmetric swelling of an upper limb or lopsided facial swelling after a few days. The use of suramina [5] drug that kills the adult worm, gives similar results, the same asymmetric limb and facial swelling. The facial distortion especially adversely affects compliance to a drug that is taken once or twice a year, Women were mortified to see their distorted faces when they looked in the mirror (Figures $1 \& 2$ ). 


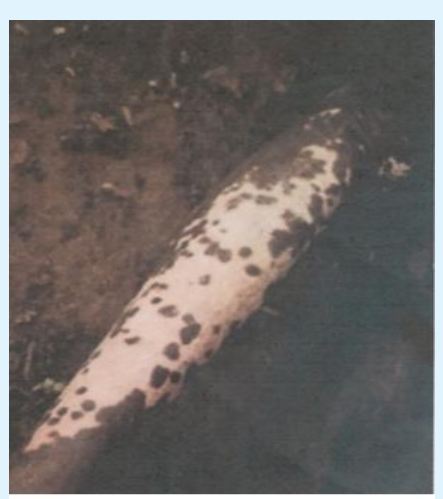

Figure 1: Extreme depigmentation- leopard skin of one leg in a 65year old onchocerciasis patient.

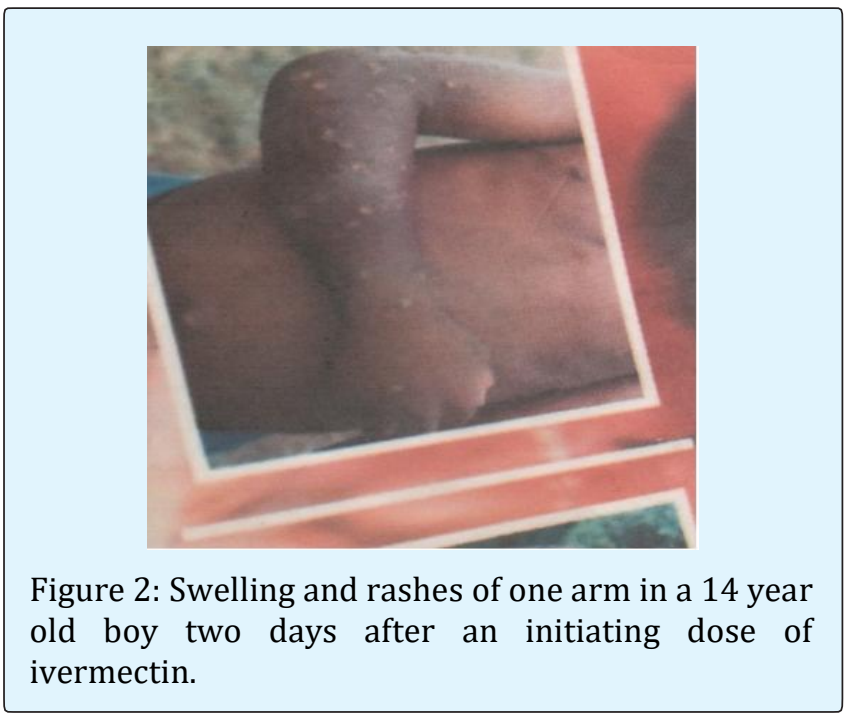

Moxidectin has been approved by US Food and Drug Administration in 2018 to replace ivermectin as drug of choice for onchocerciasis. Another filarial disease that has a devastating effect on the physical appearance of patients is transmitted by mosquitoes and the parasite is Wuchereria bancrofti. The parasite can be detected in blood but exerts its effect mostly in one lower limb, hence "elephantiasis". The groin and the scrotum may also be unilaterally and grotesquely affected (Figure3).

\section{Conclusion}

It is tempting to dismiss asymmetry as a trivial medical oddity. It is important. Disfigurement of face or body may attract stigmatization from unaffected members of the community, friends and even spouses. If disfigurement occurs after drug administration, it may deter patient from further drug intake. Although the filarial diseases were mentioned in detail, the issue of asymmetry should be studied in other diseases.

\section{References}

1. Okonkwo P, Akpa A, Ihekwaba A, Nwagbo D, Umeh $\mathrm{R}$, et al. (1991) Studies on onchocerciasis in forestsavannah mosaic areas of Nigeria. Investigations in Gbaragu, Oji River of Nigeria. Clinical manifestations and entomology. Annals of Tropical Medicine and Parasitology 65(6): 617-623.

2. Okonkwo P, Ogbuokiri JE, Ofoegbu E, Klotz U (2003) Protein binding and ivermectin estimations in patients with onchocerciasis. Clinical Pharmacology and Therapeutics 53(4): 426-430.

3. Umeh RE, Chijioke CP, Okonkwo PO (1996) Eye disease in an endemic area of the savannah forest mosaic of Nigeria. Bulletin of the World Health Organisation 74(1): 95-100.

4. Shu EN, Ogbudu SO, Ibekwe E, Okonkwo PO (1998) Nutritional status of onchocerciasis patients, an investigation of iron deficiency anaemia. Orient Journal of Medicine 10: 1-4.

5. Chijioke $\mathrm{CP}$, Umeh $\mathrm{KE}$, Mbah AU, Nwonu $\mathrm{P}$, Fleckenstein I, et al. (1998) Clinical pharmacokinetics of suramin in patients with onchocerciasis. European Journal Clinical Pharmacology 54(3): 249-251.

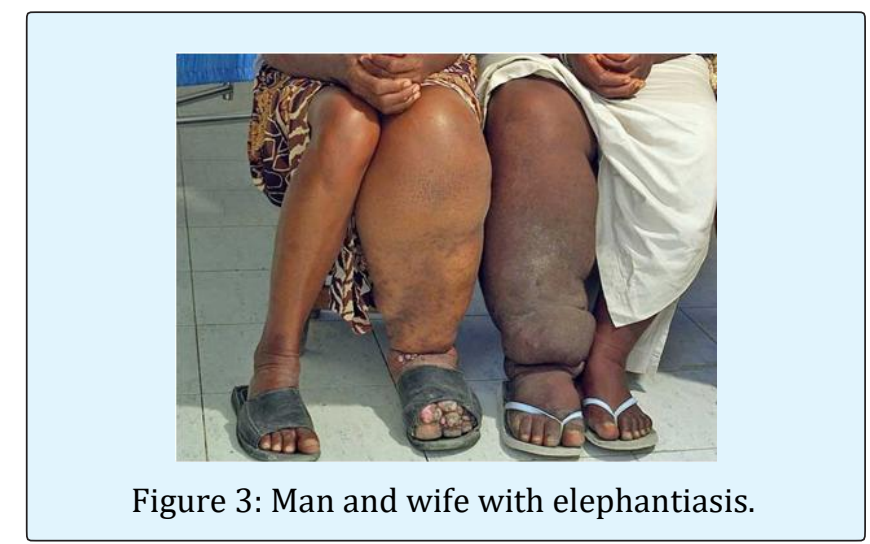

Figure 3: Man and wife with elephantiasis.

Okonkwo PO. Asymmetry in Health and Disease: Food for Thought. Epidemol Int J 2018, 2(2): 000113. 